\title{
Laparoscopic-assisted Resection for Jejunal Stenosis Following Extensive Portomesenteric Venous Thrombosis
}

\author{
Satoshi Yokoyama, MD, Daisuke Ito, Akinori Sekioka, Nao Kawaguchi, Harumi Yamada, \\ Michio Kuwahara, Hirofumi Utsunomiya, Shiro Uyama \\ Department of Surgery, Japanese Red Cross Society, Wakayama Medical Center, Wakayama, Japan (all authors).
}

\begin{abstract}
Introduction: Portomesenteric venous thrombosis (PMVT) is a rare and often fatal disease that is difficult to diagnose. The symptoms are nonspecific, and diagnosis and initial treatment tend to be delayed. The initial treatment strategy for PMVT has recently tended toward the use of conservative methods before surgical intervention, and most patients can be successfully treated in this way. In rare cases, however, bowel strictures may develop, owing to chronic ischemia.
\end{abstract}

Case Description: We present a case of elective laparoscopy-assisted small-bowel resection of ischemic jejunal stenosis following extensive portal, mesenteric, and splenic vein thrombosis.

Discussion: Laparoscopy is indicated for this condition because of the possible need for repeated operations and extensive intraoperative exploration. However, laparoscopy with pneumoperitoneum can be a risk factor for recurrent PMVT, indicating the need to develop methods to shorten the operation time.

Key Words: Delayed bowel strictures, Laparoscopic surgery, Pneumoperitoneum, Portomesenteric venous thrombosis.

\footnotetext{
Citation Yokoyama S, Ito D, Sekioka A, Kawaguchi N, Yamada H, Kuwahara M, Utsunomiya H, Uyama S. Laparoscopic-assisted resection for jejunal stenosis following extensive portomesenteric venous thrombosis. CRSLS e2014.00214. DOI: 10.4293/CRSLS.2014.00214.

Copyright $\odot 2014$ SLS This is an open-access article distributed under the terms of the Creative Commons Attribution-Noncommercial-ShareAlike 3.0 Unported license, which permits unrestricted noncommercial use, distribution, and reproduction in any medium, provided the original author and source are credited.

The authors declare no conflicts of interest.

Address correspondence to: Satoshi Yokoyama, MD, Department of Surgery, Japanese Red Cross Society, Wakayama Medical Center, 4-20 Komatsubara-dori, Wakayama, Japan. Telephone: (+81) 73422 4171, Fax: (+81) 73426 1168, E-mail: yokoyama.st@gmail.com
}

\section{INTRODUCTION}

Portomesenteric venous thrombosis (PMVT) is a rare and often fatal disease that is difficult to diagnose. ${ }^{1}$ The symptoms are nonspecific, and diagnosis and initial treatment tend to be delayed. However, once mesenteric vein thrombosis (MVT) is suspected, computed tomography (CT) should be performed. Cases with signs of peritonitis, transmural bowel infarction, or hemodynamic instability require immediate exploratory laparotomy. The initial treatment strategy for MVT has recently tended toward the use of conservative methods before surgical intervention, and most patients can be successfully treated in this way. ${ }^{2-4}$ In rare cases, however, bowel strictures may develop, owing to chronic ischemia.

We present a case of elective laparoscopy-assisted smallbowel resection of ischemic jejunal stenosis after extensive portal, mesenteric, and splenic vein thrombosis.

\section{CASE REPORT}

A 71-year-old woman was referred to our hospital with a history of diffuse epigastric pain for 7 days. She had no nausea or vomiting. One episode of melena had occurred 2 days before she presented to the emergency department. She had no significant medical history. On physical examination, her temperature was $37.8^{\circ} \mathrm{C}$. Palpation of the upper abdomen produced pain without guarding or rigidity. Laboratory data showed hemoglobin $14.6 \mathrm{mg} / \mathrm{dL}$, leukocytosis $\left(24600 / \mathrm{mm}^{3}\right)$, and significantly increased C-reactive protein $(14.19 \mathrm{mg} / \mathrm{dL})$ and D-dimer $(4.39 \mu \mathrm{g} / \mathrm{mL})$ levels, with no other significant abnormalities. Immediate contrast-enhanced CT of the abdomen revealed extensive thrombosis of the superior mesenteric vein (SMV), portal vein (PV), splenic vein (SPV), and inferior mesenteric vein (IMV). There was free fluid in the abdomen and pelvis (Figure 1). The wall of the small intestine was thickened, but there was no suggestion of intestinal necrosis (Figure 2). Laparotomy was not considered necessary. We began thrombolytic therapy. 

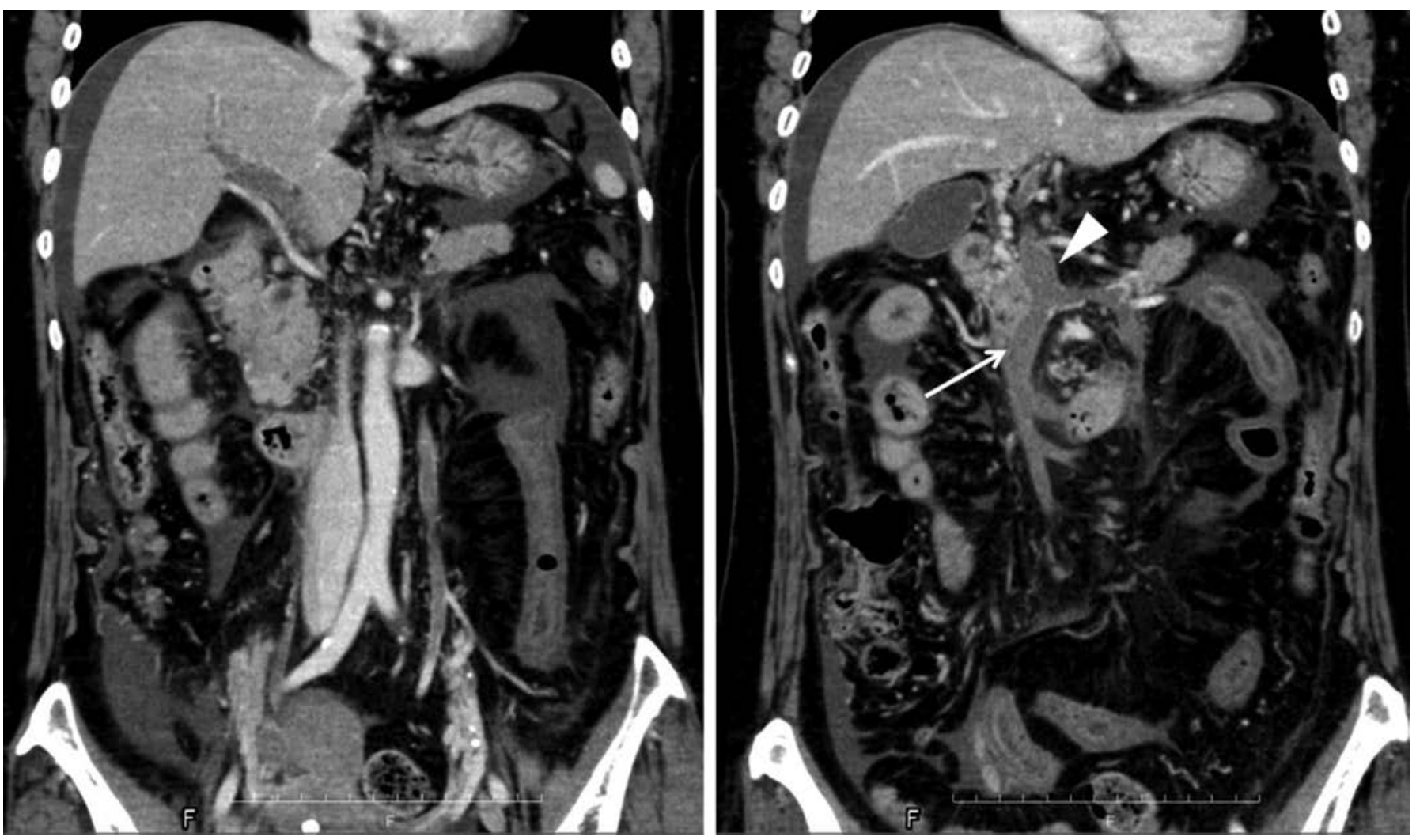

Figure 1.Abdominal CT showing extensive thrombosis of the SMV, PV, SPV, and IMV. Arrows: SMV; arrowheads: main PV.

Angiography revealed intense enhancement in the small intestine on delayed phase. There was no visualization of the SMV, SPV, IMV, or the main PV. At first, urokinase (240 000 IU) was administered as a bolus through a catheter inserted in the SMA and IMA. After that, the patient was treated with long-term thrombolysis via a catheter placed in the SMA with 240000 IU of urokinase per day for 3 days, systemic heparin (10 $000 \mathrm{IU} /$ day), and total parenteral nutrition for 14 days. A follow-up CT scan 4 days later revealed that, although the main PV and proximal SMV were not patent, blood flow was present in the distal SMV and the collateral veins. The collateral veins around the head of the pancreas from the colic vein to the left gonadal vein and to the inferior vena cava had the appearance of cavernous transformation. CT showed an improvement in the intestinal edema and the resolution of ascites. When the thrombolytic treatment was discontinued after 14 days, abdominal pain and tenderness gradually resolved. Heparin therapy was stopped and replaced by warfarin, to maintain an international normalized ratio of 2 to 3 and prevent rethrombosis. The patient was discharged from the hospital 20 days after admission. A follow-up CT scan 1 month later revealed only a small amount of residual throm-

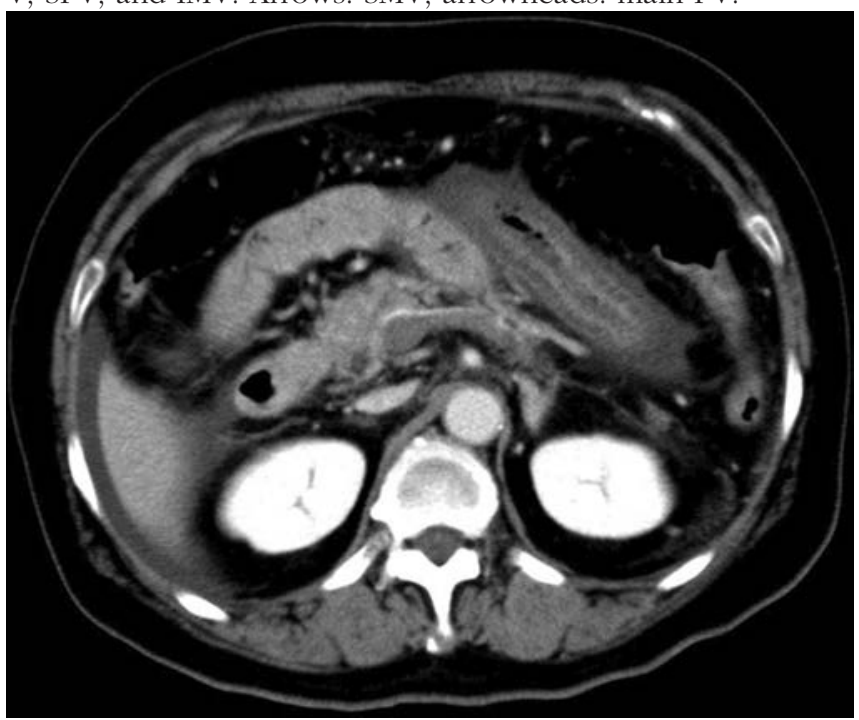

Figure 2. CT scan revealing the thickened wall of the small intestinewith no suggestion of intestinal necrosis.

botic material in the SMV. Laboratory workup did not reveal any hematologic disorders.

Six months later, the patient presented with diffuse abdominal pain. On physical examination, there was 

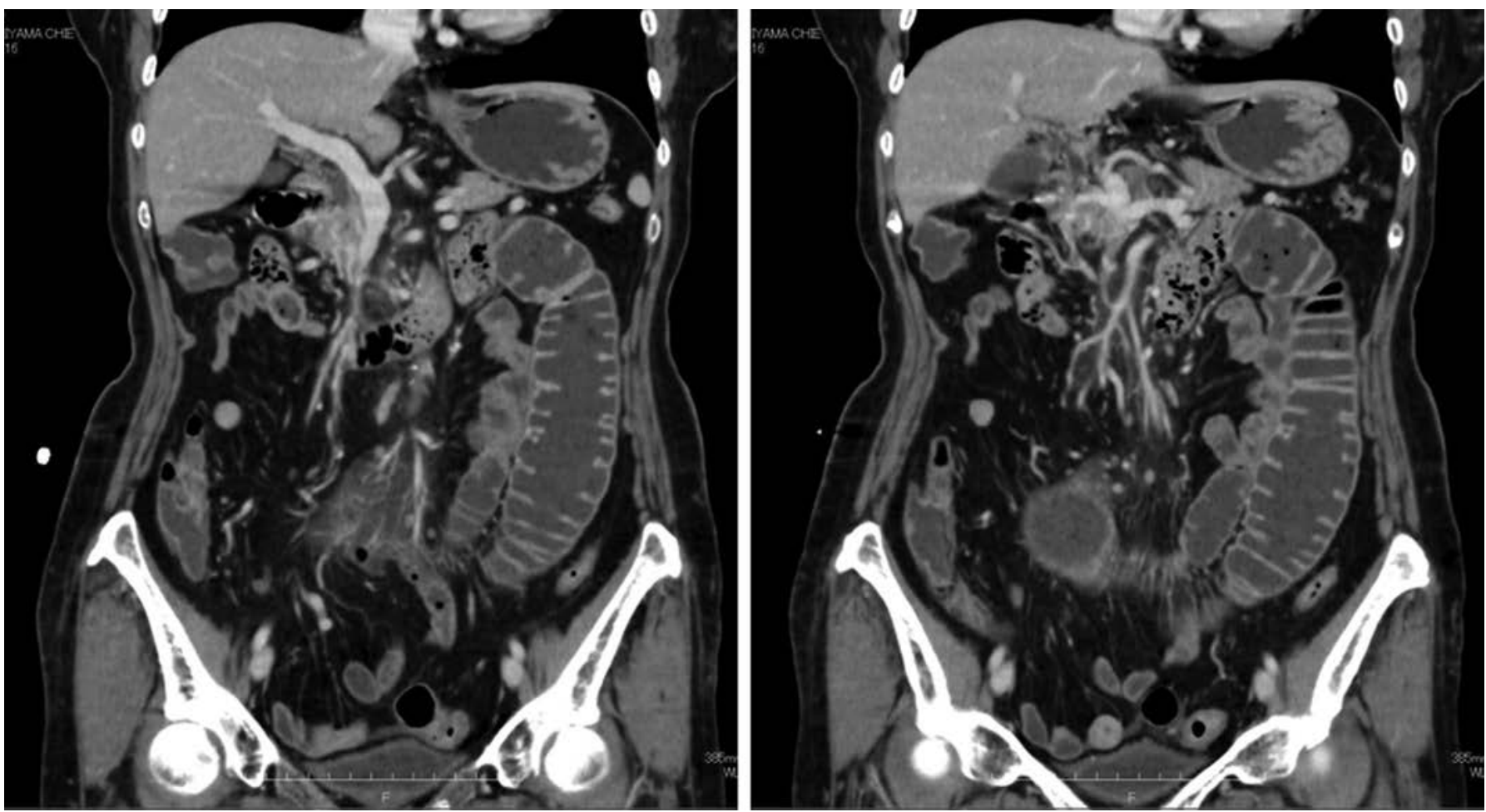

Figure 3. CT scan revealing completely patent PV, SMV, and SPV, with no evidence of cavernous transformation of the PV.

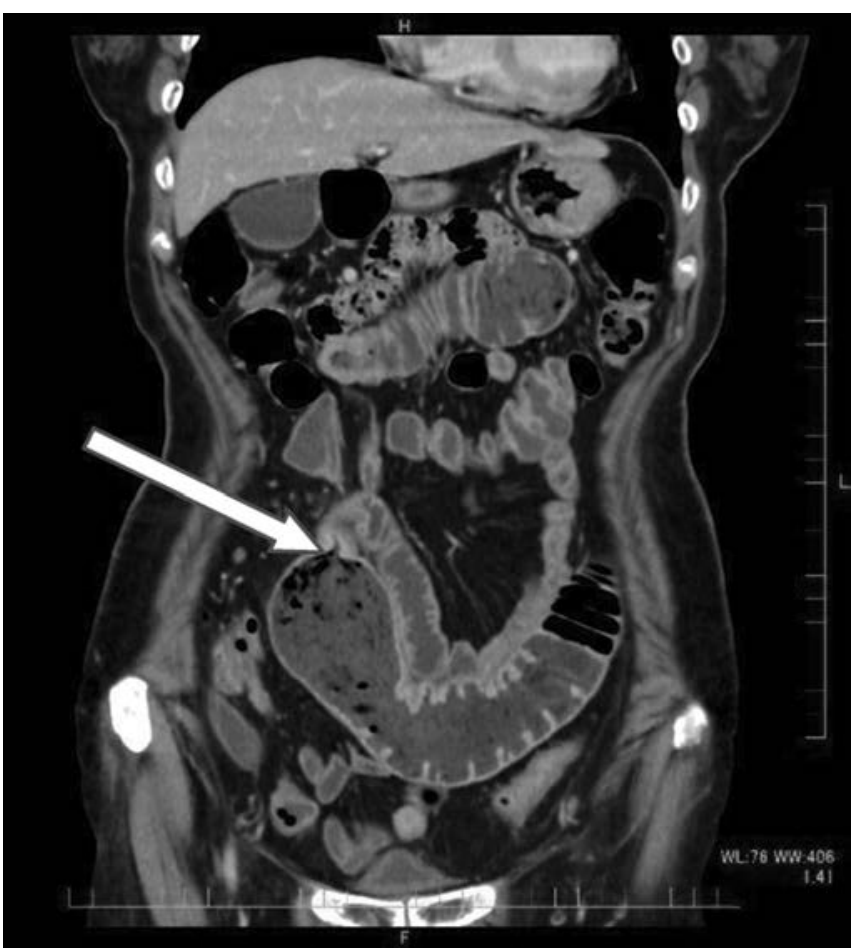

Figure 4. CT scans demonstrating severe jejunum dilatation, suspicious for postischemic small-bowel stenosis (arrow).

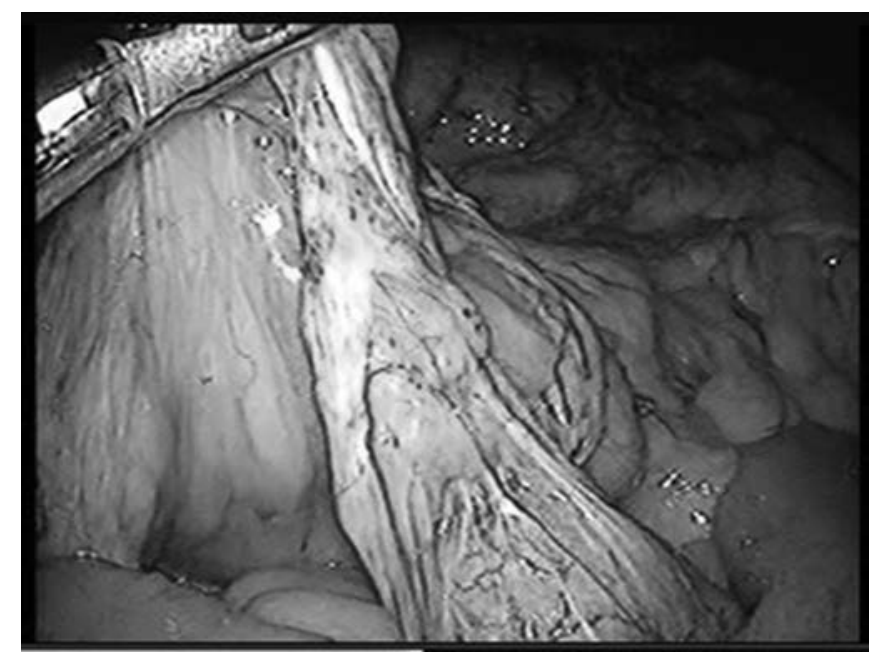

Figure 5. During laparoscopy, the stenosed segment was visibly adherent to the adjacent omentum, with associated blood vessels extending from the omentum to the segment.

abdominal tenderness in the periumbilical region without guarding or rigidity. Laboratory data were unremarkable. A CT scan revealed that the PV, SMV, and SPV were completely patent and that cavernous transformation of the PV had not occurred (Figure 3); how- 

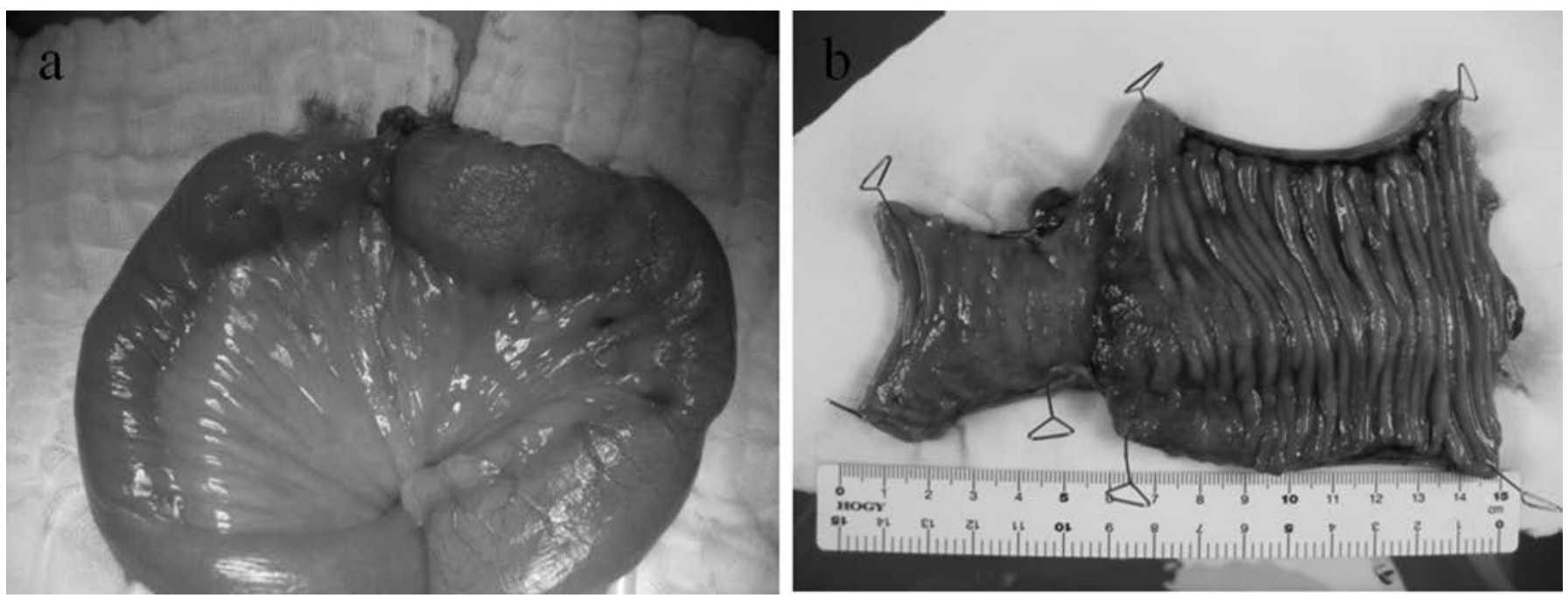

Figure 6. a, A 2-cm-long stenosed section of jejunum about $100 \mathrm{~cm}$ from the ligament of Treitz. b, Stenotic bowel segment showing a $1-\mathrm{cm}$ ulceration with severe bowel wall thickening.

ever, there was severe jejunum dilatation, suspicious for postischemia small-bowel stenosis (Figure 4).

After normalization of clotting parameters, elective laparoscopic surgery was performed 2 months after this second presentation. With the patient under general anesthesia, the open Hasson technique was used to insert a 12-mm trocar for introduction of the laparoscope via the transumbilical route. Laparoscopic inspection of the peritoneal cavity revealed adherence of the terminal ileum to the right lower abdominal wall and a $2-\mathrm{cm}-$ long stenosed section of jejunum about $100 \mathrm{~cm}$ from the ligament of Treitz, with severe dilatation of the segments proximal to it. Two 5-mm working ports were used in the right and left suprapubic regions. The stenosed segment was visibly adherent to the adjacent omentum, with associated blood vessels extending from the omentum to the segment (Figure 5). The rest of the small bowel was palpated in both directions, from the proximal jejunum to the distal ileum and vice versa and carefully inspected, but no other abnormalities were found. The stenosed jejunal segment was mobilized under laparoscopic guidance and transferred under the transumbilical trocar site (Figure 6a). The transumbilical incision was extended by $4 \mathrm{~cm}$, the small bowel and adherent omentum were delivered though the wound, and a limited small-bowel resection followed by an end-to-end anastomosis was performed in the standard fashion with a hand-sewn technique in two layers. The mesenteric defect was closed. The bowel was then returned to the abdominal cavity. Inspection of the stenotic bowel segment showed a 1-cm ulceration with severe bowel wall thickening (Figure 6b). Histopathologic examination showed a 1-cm ulceration, fibrosis, and granulation tissue disrupting the architecture of the muscular fibers and thrombosis of the vessels with some recanalization.

The postoperative course was uneventful. The patient was given heparin. Oral feeding was started on the second postoperative day, and the heparin therapy was replaced by warfarin. The patient was discharged 5 days after the operation and, at this writing, has remained well and without symptoms for 6 months.

\section{DISCUSSION}

PMVT is an uncommon, potentially lethal condition that accounts for $5 \%$ to $15 \%$ of all mesenteric ischemic events. ${ }^{1}$ However, with advances in diagnostic imaging modalities and treatment, an increasing number of cases of PMVT successfully treated with conservative therapy have been reported. ${ }^{2-4}$ It is very important to establish a strategy for the efficient management of late sequelae occurring after successful conservative treatment of this condition. Ischemic bowel stenosis is one of the most frequent late sequelae of PMVT. ${ }^{5-8}$ Once PMVT develops, it causes extensive bowel congestion and renders parts of the intestine vulnerable to thromboembolism or arterial spasm, resulting in bowel wall ischemia, which can cause multiple latent infarctions or persistent circulatory disturbance, despite successful conservative treatment. Because ischemic bowel stenosis is caused by latent infarctions or persistent circulatory disturbance, it can occur in multiple locations at the same time or serially.

The role of laparoscopy in the diagnosis of mesenteric ischemia is not yet fully established. However, considering that 
delay in diagnosis and surgical exploration is still frequent and is a significant contributory factor to the reported high mortality rate, diagnostic laparoscopy in an early position in the management algorithm for acute MVT can furnish a rapid precise diagnosis of bowel infarction. It can also reduce the unnecessary laparotomies in difficult cases. ${ }^{?}$

Laparoscopy is considered to be the standard of care for this condition. In particular, it is very effective for extensive exploration of potentially stenotic sites, in that it can be performed with a much smaller incision and is less likely to cause adhesions than an open operation. ${ }^{10-12}$ Laparoscopy is thus effective in cases of metachronous multiple stenoses necessitating repeated surgeries.

It has been reported that pneumoperitoneum during laparoscopic procedures can decrease intra-abdominal blood perfusion ${ }^{13-15}$ and thereby act as a cause of PMVT. ${ }^{16}$ In surgical planning for our case, we considered using gasless abdominal wall lifting instead of pneumoperitoneum, to avoid decreasing intra-abdominal blood flow. ${ }^{17-19}$ However, the gasless abdominal wall-lifting method is suboptimal for diagnostic laparoscopy because it is more likely to result in a narrower laparoscopic field of view than pneumoperitoneum allows.

To maintain operative safety, we applied the following ideas to shorten the duration of pneumoperitoneum: First, we did not hesitate to insert an additional port if the laparoscopic view was insufficient for diagnosis or operative procedures, and second, we used a laparoscopy assisted approach with a small laparotomy, because a completely laparoscopic approach often requires longer operative time. ${ }^{20} \mathrm{~W}$ ith these precautions, we completed the operation in 66 minutes. If subsequent bowel stenoses were to develop, we feel that a second operation could be performed safely with this approach.

In conclusion, we present a case of ischemic bowel stenosis that occurred after treated conservatively PMVT. Laparoscopy is indicated for this condition because of the possible need for repeated operations and extensive intraoperative exploration. However, laparoscopy using pneumoperitoneum can be a risk factor for recurrent PMVT, indicating the need to develop methods to shorten the operative time.

\section{References:}

1. Grendell JH, Ockner RK. Mesenteric venous thrombosis. Gastroenterology. 1982;82:358-372.

2. Kumar S, Sarr MG, Kamath PS. Mesenteric venous thrombosis. N Engl J Med. 2001;345:1683-1688.

3. Brunaud L, Antunes L, Collinet-Adler S, et al. Acute mesenteric venous thrombosis: case for nonoperative management. $J$ Vasc Surg. 2001;34:673-679.
4. Cenedese A, Monneuse O, Gruner L, et al. Initial management of extensive mesenteric venous thrombosis: retrospective study of nine cases. World J Surg. 2009;33:2203-2208.

5. Eugène C, Valla D, Wesenfelder L, et al. Small intestinal stricture complicating superior mesenteric vein thrombosis: a study of three cases. Gut. 1995;37:292-295.

6. Antoch G, Hansen O, Pourhassan S, et al. Ischaemic jejunal stenosis complicating portal and mesenteric vein thrombosis: a report of two cases. Eur J Gastroenterol Hepatol. 2001;13:707-710.

7. Huh SH, Kim DI, Lee BB. Superior mesenteric thrombosis associated with small bowel stricture. J Cardiovasc Surg (Torino). 2002; 43:895-897.

8. Yang J, Shen L, Zheng X, et al. Small bowel stricture complicating superior mesenteric vein thrombosis. J Huazhong Univ Sci Technol Med Sci. 2012;32:146-148.

9. Zamir G, Reissman P. Diagnostic laparoscopy in mesenteric ischemia. Surg Endosc. 1998;12:390-393.

10. O'Connor DB, Winter DC. The role of laparoscopy in the management of acute small-bowel obstruction: a review of over 2,000 cases. Surg Endosc. 2012;26:12-17.

11. Cirocchi R, Abraha I, Farinella E, et al. Laparoscopic versus open surgery in small bowel obstruction. Cochrane Database Syst Rev. 2010;17:CD007511.

12. Ghosheh B, Salameh JR. Laparoscopic approach to acute small bowel obstruction: review of 1061 cases. Surg Endosc. 2007;21:1945-1949.

13. Jakimowicz J, Stultiëns G, Smulders F. Laparoscopic insufflation of the abdomen reduces portal venous flow. Surg Endosc. 1998;12:129-132.

14. Odeberg S, Ljungqvist O, Sollevi A. Pneumoperitoneum for laparoscopic cholecystectomy is not associated with compromised splanchnic circulation. Eur J Surg. 1998;164:843-848.

15. Takagi S. Hepatic and portal vein blood flow during carbon dioxide pneumoperitoneum for laparoscopic hepatectomy. Surg Endosc. 1998;12:427-431.

16. James AW, Rabl C, Westphalen AC, et al. Portomesenteric venous thrombosis after laparoscopic surgery: a systematic literature review. Arch Surg. 2009;144:520-526.

17. Huang CC, Yang CY, Wu MH, et al. Gasless laparoscopyassisted versus open resection of small bowel lesions. J Laparoendosc Adv Surg Tech A. 2010;20:699-703.

18. Han C, Ding Z, Fan J, et al. Comparison of the stress response in patients undergoing gynecological laparoscopic surgery using carbon dioxide pneumoperitoneum or abdominal wall-lifting methods. $J$ Laparoendosc Adv Surg Tech A. 2012;22:330-335.

19. Hyodo M, Sata N, Koizumi M, et al. Laparoscopic splenectomy using pneumoperitoneum or gasless abdominal wall lifting: a 15-year single institution experience. Asian J Endosc Surg. 2012;5:63-68.

20. Ehrmantraut W, Sardi A. Laparoscopy-assisted small bowel resection. Am Surg. 1997;63:996-1001. 